\title{
Acidentes de trabalho em segmentos do agronegócio paranaense
}

\author{
Accidents at work in segments of Paraná agribusiness \\ Accidentes de trabajo en segmentos de la agroindustria paranaense
}

Recebido: 05/02/2021 | Revisado: 09/02/2021 | Aceito: 12/02/2021 | Publicado: 19/02/2021

\author{
Jéssica Cristina Ruths \\ ORCID: https://orcid.org/0000-0002-7400-1191 \\ Universidade Federal do Paraná, Brasil \\ E-mail: ruthsjessica@gmail.com \\ Marcos Paulo Rodrigues de Souza \\ ORCID: https://orcid.org/0000-0002-4212-9895 \\ Universidade Estadual do Paraná, Brasil \\ E-mail: marcos.paulo@unespar.edu.br \\ Jefferson Andronio Ramundo Staduto \\ ORCID: https://orcid.org/0000-0003-1855-1292 \\ Universidade Estadual do Oeste do Paraná, Brasil \\ E-mail: jstaduto@yahoo.com.br \\ Carla Maria Schmidt \\ ORCID: https://orcid.org/0000-0001-8364-2663 \\ Universidade Estadual do Paraná, Brasil \\ E-mail: carlamariaschmidt@hotmail.com
}

\begin{abstract}
Resumo
Acidentes de trabalho se vinculam a diversificação dos processos produtivos e ao perfil de produção, logo pesquisas sobre o tema devem considerar as especificidades das atividades envolvidas. Apesar do número crescente de trabalhos sobre a temática, ainda há carência de estudos que avaliem especificamente o setor do agronegócio, portanto, objetivouse analisar os acidentes de trabalho nos segmentos de insumos, primário e agroindustrial do agronegócio, notificados no Paraná, no período de 2012 a 2017. Desenvolveu-se um estudo epidemiológico, descritivo e quantitativo, com base nas Comunicações de Acidentes de Trabalho (CAT), registradas no Instituto Nacional de Seguridade Social (INSS). Foram comunicadas 51.071 CAT relacionadas ao agronegócio no Estado, a taxa de incidência foi de 72,73 acidentes a cada mil trabalhadores. No período estudado, houve diminuição do número de CAT em todos os segmentos analisados. Destacaram-se os acidentes de trabalho no abate de suínos, aves e outros pequenos animais, foram 12.923 CAT registadas. A ocupação mais vulnerável foi a de alimentador de linha de produção, com 10.230 acidentes. A parte do corpo mais atingida foram os dedos 15.907 (31,15\%), e, dentre os agentes causadores, as facas e fações estavam entre os principais, causando $4.782(9,36 \%)$ lesões. Este estudo reforça a importância da análise dos acidentes de trabalho no agronegócio paranaense, de modo que, espera-se contribuir com evidências que auxiliem na prevenção destes agravos nos segmentos analisados.
\end{abstract}

Palavras-chave: Agroindústria; Notificação de acidentes de trabalho; Trabalhadores rurais.

\begin{abstract}
Accidents at work are linked to the diversification of productive processes and to the production profile, so research on the subject must consider the specificities of the activities involved. Despite the growing number of studies on the topic, there is still a lack of studies that specifically evaluate the agribusiness sector, therefore, this study aimed to analyze occupational accidents in the input, primary and agroindustrial agribusiness segments, reported in Paraná from 2012 to 2017. An epidemiological, descriptive and quantitative study was developed, based on the Communications of Work Accidents (CAT), registered with the National Institute of Social Security (INSS). There were 51,071 CAT related to agribusiness in the state, the incidence rate was 72.73 accidents per thousand workers. In the period studied, there was a decrease in the number of CAT in all the segments analyzed. The work accidents in the slaughtering of pigs, poultry and other small animals stood out, there were 12,923 CAT registered. The most vulnerable occupation was that of production line feeder, with 10,230 accidents. The most affected body part was the fingers 15,907 (31.15\%), and, among the causative agents, knives and fables were among the main ones, causing 4,782 (9.36\%) injuries. This study reinforces the importance of the analysis of work accidents in the agribusiness of Paraná, so that it is expected to contribute with evidence to help prevent these injuries in the analyzed segments.
\end{abstract}

Keywords: Agribusiness; Notification of accidents at work; Rural workers.

\section{Resumen}

Los accidentes laborales están vinculados a la diversificación de los procesos de producción y al perfil productivo, por lo que la investigación sobre el tema debe tener en cuenta las especificidades de las actividades implicadas. A pesar del creciente número de estudios sobre el tema, aún faltan estudios que evalúen específicamente el sector del agronegocio, 
por lo tanto, este estudio tuvo como objetivo analizar los accidentes de trabajo en los segmentos de insumos, primario y agroindustrial del agronegocio, reportados en Paraná, desde 2012 hasta 2017. Se desarrolló un estudio epidemiológico, descriptivo y cuantitativo a partir de las Comunicaciones de Accidentes de Trabajo (CAT) registradas en el Instituto Nacional de la Seguridad Social (INSS). Hubo 51.071 CAT relacionados con la agroindustria en el Estado, el índice de incidencia fue de 72,73 accidentes por cada mil trabajadores. En el periodo estudiado, se produjo una disminución del número de CAT en todos los segmentos analizados. Destacan los accidentes de trabajo en el sacrificio de cerdos, aves de corral y otros animales pequeños; se registraron 12.923 CAT. La ocupación más vulnerable fue la de alimentador de línea de producción, con 10.230 accidentes. La parte del cuerpo más afectada fueron los dedos 15.907 (31,15\%), y, entre los agentes causantes, los cuchillos y las fábulas estuvieron entre los principales, causando 4.782 (9,36\%) lesiones. Este estudio refuerza la importancia de analizar los accidentes de trabajo en la agroindustria de Paraná, por lo que se espera contribuir con evidencias que ayuden a prevenir estas lesiones en los segmentos analizados.

Palabras clave: Agroindustria; Notificación de accidentes de trabajo; Trabajadores rurales.

\section{Introdução}

O Paraná tem se destacado no agronegócio em função dos elevados índices de produtividade e exportação de itens agropecuários (Felema et al., 2013). Em 2017, os produtos rurais foram responsáveis por 14,20\% das exportações nacionais (Governo Federal, 2017). O Estado lidera a produção de carne e produtos florestais, é o segundo maior produtor de soja e se destaca no cultivo de cereais e produtos do complexo sucroenergético (Governo Federal, 2017; Prestes et al., 2018).

O setor possui também um papel importante na geração de renda e emprego. O Valor Bruto da Produção (VBP) agropecuária totalizou R\$ 89,8 bilhões em 2018, houve crescimento de 5\% em relação a 2017 e havia 556.774 pessoas formalmente ocupadas no agronegócio (Governo Federal, 2017; Ministério do Trabalho, 2020). Contudo, seu volume de produção e número de empregos refletem na proporção dos acidentes de trabalho.

Historicamente os acidentes ocorridos durante as atividades ocupacionais se relacionam com fatores sociais, econômicos, tecnológicos e organizacionais, vinculando-se a diversificação dos processos produtivos e ao perfil de produção (Moreira et. al., 2015). São considerados acidentes de trabalho àqueles que ocorrem devido ao desenvolvimento do trabalho, a serviço de empresa ou de empregadores domésticos e que podem provocar a morte do trabalhador, perturbação funcional ou lesão corporal, de forma permanente ou temporária (Governo Federal, 2017).

Em 2018, ocorreram 48,8 mil acidentes de trabalho no Paraná, 197 com óbito. O Estado foi o quarto em termos de proporção de notificações no país (OSST, 2018), cenário que motivou a escolha do mesmo como campo do presente estudo. No mesmo ano, o setor com maior número de notificações não letais foi o de abate de suínos, aves e outros animais pequenos com 3,3 mil notificações, seguido pelas atividades de atendimento hospitalar e pelo comércio varejista de mercadorias em geral, com 3,06 mil e 1,6 mil notificações, respectivamente (OSST, 2018).

Os acidentes de trabalho, em virtude da sua relevância epidemiológica, são considerados assuntos de grande importância em pesquisas de saúde pública e de mercado de trabalho (Ultramari et al., 2012). Estudos sobre esse tema devem levar em consideração as especificidades das atividades envolvidas, pois o processo de trabalho envolve características físicas, biológicas, químicas, ergonômicas e mecânicas distintas (Moreira et al., 2015), podendo impactar diferencialmente o perfil dos acidentes. Apesar do número crescente de trabalhos sobre a temática, ainda há carência de estudos que avaliem especificamente o setor do agronegócio, assim, objetivou-se analisar acidentes de trabalho nos segmentos de insumo, primário e agroindustrial do agronegócio, notificados no Paraná, no período de 2012 a 2017, utilizando indicadores de acidentes de trabalho oriundos das Comunicações de Acidentes de Trabalho (CAT), registradas no Instituto Nacional de Seguridade Social (INSS).

Buscou-se identificar e dimensionar as principais lesões ocorridas, os agentes causadores, as atividades econômicas e ocupações acometidas, a fim de construir uma base de conhecimento específica a respeito de grupos vulneráveis e enriquecer o debate para a área temática, além de apresentar dados empiricamente relevantes que possam auxiliar na elaboração de políticas públicas e ações de prevenção de acidentes de trabalho no agronegócio. 


\section{Metodologia}

Desenvolveu-se um estudo epidemiológico, descritivo e quantitativo. O campo do estudo é o Paraná, que conta com 399 municípios distribuídos em uma área de 199.299 km² e população estimada de 11.516 .840 pessoas (IBGE, 2020). Neste Estado, a agricultura, a pecuária, a indústria e os serviços são as principais atividades socioeconômicas (IBGE, 2019).

A população do estudo foi composta por trabalhadores dos segmentos de insumos, primário, e da agroindústria do agronegócio do Paraná, que sofreram acidentes de trabalho registrados em CAT, no período de 2012 a 2017. A seleção temporal se deve ao estado de completude do banco de dados em razão da atualização dos registros da CAT. As atividades econômicas relacionados ao agronegócio foram selecionadas e analisadas por segmentos do setor, exceto para o de agrosserviços, de acordo com a metodologia elaborada pelo Centro de Estudos Avançados em Economia Aplicada (CEPEA) (Tabela 1). O CEPEA (2017) define o agronegócio como um conjunto de atividades econômicas relacionadas, direta ou indiretamente, à agropecuária.

Tabela 1. Atividades e segmentos do agronegócio selecionados e respectivas Classificações Nacionais de Atividades Econômica (CNAEs) (2.0).

\begin{tabular}{lll}
\hline CNAE 2.0 & Segmento & Atividade \\
\hline 2012 e 2013 & Insumos & Fertilizantes e corretivos de solo \\
20517 & Insumos & Defensivos agrícolas \\
10660 & Insumos & Rações \\
21220 & Insumos & Medicamentos veterinários \\
283 & Insumos & Máquinas para a agropecuária \\
\hline 011 a 014 e 02 & Primário & Agricultura e floresta \\
$015 ; 017$ e 03 & Primário & Pecuária, pesca e aquicultura \\
\hline 101 e 102 & Agroindústria & Abate e preparação de carnes e pescados \\
105 & Agroindústria & Laticínios \\
107 e 193 & Agroindústria & Açúcar e etanol \\
108 & Agroindústria & Indústria do café \\
103 & Agroindústria & Fabricação de conserva de frutas, legumes e outros vegetais \\
104 & Agroindústria & Fabricação de óleos e gorduras vegetais e animais \\
106 (exceto 10660$)$ & Agroindústria & Moagem, fabricação de produtos amiláceos (exceto para animais) \\
109 & Agroindústria & Outros produtos alimentares \\
11 & Agroindústria & Bebidas \\
12 & Agroindústria & Fabricação de produtos do fumo \\
$1311 ; 1312 ; 1321$ e 1322 & Agroindústria & Têxtil de base natural \\
14 & Agroindústria & Vestuário e acessórios \\
$1510 ; 1529 ; 1531$ & Agroindústria & Artigos de couro e calçados \\
16 & Agroindústria & Fabricação de produtos de madeira \\
17 & Agroindústria & Fabricação de celulose, papel e produtos de papel \\
3101 & Agroindústria & Móveis de madeira \\
$46 ; 47 ; 49$ a $53 ; 55 ; 56 ; 58$ & Agrosserviços & Diversos \\
a 66; 68 a $75 ; 77$ a 82 e 84 & & \\
\hline
\end{tabular}

Fonte: Adaptado do CEPEA (2017).

Dados sobre a CAT, foram coletados no Observatório de Segurança e Saúde no Trabalho (OSST), uma iniciativa do Ministério do Trabalho e Emprego (MTE) em cooperação com a Organização Internacional do Trabalho (OIT). As bases de dados utilizadas pelo observatório são de domínio público, sendo disponibilizadas pelos seguintes órgãos: i) Instituto Brasileiro de Geografia e Estatística (IBGE); ii) Instituto Nacional de Seguridade Social (INSS) (CATWEB); iii) Sistema Único de Informações de Benefícios da Previdência Social (SISBEN); e iv) Sistema Nacional de Agravos de Notificações (SINAN) (OSST, 2018).

A fim de obter o perfil dos acidentados, foram selecionadas as variáveis: ano de ocorrência do acidente, município do empregador, Classificação Nacional de Atividade Econômica (CNAE) do acidentado, tipo de acidente, ocupação do acidentado, 
agente causador do acidente, natureza da lesão, parte do corpo atingida e sexo do trabalhador. Já o número de trabalhadores ocupados no agronegócio foi coletado na Relação Anual de Informações Sociais (RAIS), divulgados pelo MTE.

Os dados foram tabulados em planilha no Excel Microsoft Windows 2010, ordenados e analisados por meio de estatística descritiva. Além disso, calculou-se a taxa de incidência de acidentes de trabalho do agronegócio, por segmentos. Este é um indicador da intensidade com que acontecem os acidentes de trabalho, pois expressa a relação entre as condições de trabalho e o quantitativo médio de trabalhadores expostos àquelas condições (Ministério da Economia, 2020).

A fórmula desse indicador se baseia no método descrito em Chagas et al. (2012) para indicadores da saúde. Os autores recomendam para o numerador o uso de todas as ocorrências registradas em determinado acidente e para o denominador a média da quantidade de contribuintes, com carteira assinada, para as atividades analisadas. A variável calculada baseou-se na seguinte equação:

$$
I A T=\frac{\text { número de casos de novos acidentes de trabalho }}{\text { número médio anual de vínculados }} * 1000
$$

Em que IAT significa Incidência de Acidentes de Trabalho. A IAT foi ainda trabalhada no software QGIS (Devolopment Team), para elaboração de mapa temático com intuito de analisar a espacialização deste indicador no Paraná.

A pesquisa foi baseada exclusivamente em banco de dados de acesso público. Os acidentados tampouco foram entrevistados para coleta de informações adicionais, assim, foi dispensada a submissão para apreciação por Comitê de Ética em Pesquisa, em consonância com a Resolução nº 510/2016 do Conselho Nacional de Saúde (CNS), que trata sobre os princípios da ética em pesquisa com seres humanos.

\section{Resultados}

No agronegócio paranaense, exceto no segmento de agrosserviços, entre os anos de 2012 a 2017, registraram-se 51.071 CAT, representando 22,06\% das comunicações do Estado, em 254 casos houve óbito. Entre os registros de acidentes do setor, $73,99 \%$ foram em homens e 26,01\% em mulheres, 30,67\% ocorreram com trabalhadores que tinham idade entre os 26 a 35 anos, enquanto $28,26 \%$ tinham idade entre 15 a 25 anos. O tipo de acidente mais comum foi o típico, ou seja, 85,02\% dos acidentes ocorreram a durante o exercício do trabalho para a empresa, 11,90\% foram de trajeto, pois aconteceram no caminho entre a residência e o local de trabalho, 2,89\% eram doenças do trabalho e em apenas $0,19 \%$ dos casos não havia informação sobre o tipo de acidente (Tabela 2). 
Tabela 2. Caracterização das CAT do agronegócio, segundo sexo, idade, tipo de acidente e ocorrência de óbito, 2012 a 2017 , Paraná - Brasil.

\begin{tabular}{lll}
\hline Sexo & $\mathbf{N}$ & $\mathbf{\%}$ \\
\hline Feminino & 13285 & 26,01 \\
Masculino & 37785 & 73,99 \\
Não informado & 1 & 0,002 \\
Total & 51071 & 100 \\
\hline Idade & $\mathbf{N}$ & $\mathbf{\%}$ \\
\hline Menor de 15 anos & 9 & 0,02 \\
15 a 25 anos & 14436 & 28,27 \\
26 a 35 anos & 15666 & 30,67 \\
36 a 45 anos & 11758 & 23,02 \\
46 a 55 anos & 7077 & 13,86 \\
56 a 65 anos & 1975 & 3,87 \\
Mais de 65 anos & 150 & 0,29 \\
Total & 51071 & 0,02 \\
\hline Tipo de acidente & $\mathbf{N}$ & $\mathbf{\%}$ \\
\hline Doença & 1474 & 2,89 \\
Ignorado & 98 & 0,19 \\
Típico & 43421 & 85,02 \\
Trajeto & 6078 & 11,90 \\
Total & 51071 & 100 \\
\hline Ocorrência de óbito & $\mathbf{N}$ & $\mathbf{\%}$ \\
\hline Não & 50817 & 99,50 \\
Sim & 254 & 0,50 \\
Total & 51071 & 100 \\
\hline
\end{tabular}

Fonte: Autores com base em dados do OSST (2018).

Entre os segmentos analisados, a agroindústria apresentou o maior número das comunicações de acidente de trabalho $82,45 \%$, seguida pelo segmento primário $14,77 \%$ e de insumos 2,78\% (Tabela 3). Percebe-se que entre os anos de 2012 a 2017 houve diminuição do número de acidentes em todos os segmentos analisados. Nesse período o segmento que apresentou maior variação foi o de insumos $-27,45 \%$, seguido pelo primário $-15,57 \%$ e agroindústria $-13,97 \%$ (Tabela 3 ).

Tabela 3. Frequência e percentual de CAT do agronegócio segundo o ano, 2012 a 2017, Paraná - Brasil.

\begin{tabular}{lllllllll}
\hline & \multicolumn{2}{c}{ Agroindústria } & \multicolumn{2}{c}{ Insumos } & \multicolumn{2}{c}{ Primário } & \multicolumn{2}{c}{ Agronegócio } \\
\hline \multicolumn{1}{c}{ Ano } & $\mathbf{N}$ & $\mathbf{\%}$ & $\mathbf{N}$ & $\mathbf{\%}$ & $\mathbf{N}$ & $\mathbf{\%}$ & $\mathbf{N}$ & \% \\
\hline 2012 & 7.332 & 14,36 & 255 & 0,50 & 1.317 & 2,58 & 8.904 & 17,43 \\
2013 & 7.384 & 14,46 & 267 & 0,52 & 1.356 & 2,66 & 9.007 & 17,64 \\
2014 & 7.704 & 15,08 & 282 & 0,55 & 1.229 & 2,41 & 9.215 & 18,04 \\
2015 & 7.134 & 13,97 & 244 & 0,48 & 1.247 & 2,44 & 8.625 & 16,89 \\
2016 & 6.248 & 12,23 & 186 & 0,36 & 1.281 & 2,51 & 7.715 & 15,11 \\
2017 & 6.308 & 12,35 & 185 & 0,36 & 1.112 & 2,18 & 7.605 & 14,89 \\
\hline Total & 42.110 & 82,45 & 1.419 & 2,78 & 7.542 & 14,77 & 51.071 & 100,00 \\
\hline
\end{tabular}

Fonte: Autores com base em dados do OSST (2018).

A atividade econômica que mais apresentou CAT foi o abate de suínos, aves e outros pequenos animais com 25,30\% das notificações, já a ocupação mais frequente foi a de alimentador de linha de produção, com 20,04\% dos registros (Tabela 4). 
Tabela 4. CAT registras por segmento do agronegócio, segundo principais atividades econômicas e ocupações, 2012 a 2017, Paraná - Brasil.

\begin{tabular}{|c|c|c|c|}
\hline \multicolumn{2}{|l|}{ Segmento } & $\mathbf{N}$ & $\%^{1}$ \\
\hline \multicolumn{4}{|c|}{ Atividade econômica (CNAE) } \\
\hline Agroindústria & Abate de suínos, aves e outros pequenos animais & 12.923 & 25,30 \\
\hline Agroindústria & Desdobramento de madeira & 2.834 & 5,55 \\
\hline Agroindústria & Fabricação de móveis com predominância de madeira & 2.737 & 5,36 \\
\hline Agroindústria & Fabricação de açúcar em bruto & 2.626 & 5,14 \\
\hline Agroindústria & $\begin{array}{l}\text { Fabricação de madeira laminada e de chapas de } \\
\text { madeira compensada, prensada e aglomerada }\end{array}$ & 1.765 & 3,46 \\
\hline Todos $^{2}$ & Demais atividades econômicas & 28.186 & 44,81 \\
\hline Agronegócio & Total & 51.071 & 100 \\
\hline \multicolumn{4}{|l|}{ Ocupações (CBO) } \\
\hline Agroindústria e Insumos & Alimentador de linha de produção & 10.230 & 20,04 \\
\hline Agroindústria e Primário & Abatedor & 3.166 & 6,20 \\
\hline Primário & Trabalhador da cultura de cana-de-açúcar & 3.099 & 6,07 \\
\hline Agroindústria e Primário & Magarefe & 2.265 & 4,44 \\
\hline Todos & Mecânico de manutenção de máquinas em geral & 1.471 & 2,88 \\
\hline Todos & Demais ocupações & 30.840 & 60,39 \\
\hline Agronegócio & Total & 51.071 & 100 \\
\hline
\end{tabular}

${ }^{1}$ Porcentagem calculada em relação ao total dos acidentes do agronegócio, com exceção do segmento de agrosserviços.

${ }^{2}$ Todos: segmento de insumos, primário e agroindustrial.

Fonte: Autores com base em dados do OSST (2018).

As lesões mais comumente registradas foram corte, laceração, feridas contusas e puncturas, que representaram 26,47\% dos casos. A parte do corpo mais atingida foi o dedo, atingido em 31,15\% dos acidentes, enquanto os principais agentes causadores foram facas e facões $9,36 \%$ (Tabela 5). 
Tabela 5. CAT por segmentos do agronegócio, segundo principais lesões, partes do corpo atingidas e agentes causadores, 2012 a 2017, Paraná - Brasil.

\begin{tabular}{|c|c|c|c|}
\hline Segmento & & $\mathbf{n}$ & $\%^{1}$ \\
\hline \multicolumn{4}{|c|}{ Natureza da Lesão } \\
\hline $\operatorname{Todos}^{2}$ & Corte, laceração, ferida contusa e punctura & 13.521 & 26,47 \\
\hline Todos & Fratura & 9.062 & 17,74 \\
\hline Todos & Contusão e esmagamento & 8.941 & 17,51 \\
\hline Todos & Lesão imediata & 4.582 & 8,97 \\
\hline Todos & Distensão e torção & 3.310 & 6,48 \\
\hline Todos & Demais naturezas das lesões & 11.655 & 22,82 \\
\hline Agronegócio & Total & 51.071 & 100 \\
\hline \multicolumn{4}{|c|}{ Partes do corpo atingidas } \\
\hline Todos & Dedo & 15.907 & 31,15 \\
\hline Todos & Mão (exceto punho ou dedos) & 4.041 & 7,91 \\
\hline Todos & Pé (exceto artelhos) & 3.414 & 6,68 \\
\hline Todos & Perna & 2.824 & 5,53 \\
\hline Todos & Cabeça & 2.756 & 5,40 \\
\hline Todos & Demais partes do corpo & 22.129 & 43,33 \\
\hline Agronegócio & Total & 51.071 & 100 \\
\hline \multicolumn{4}{|c|}{ Agentes Causadores } \\
\hline $\begin{array}{l}\text { Agroindústria e } \\
\text { Primário }\end{array}$ & Faca e facão & 4.782 & 9,36 \\
\hline Todos & Máquina & 3.360 & 6,58 \\
\hline Todos & Veículo & 3.105 & 6,08 \\
\hline Todos & Madeira & 2.803 & 5,49 \\
\hline Todos & Motocicleta e motoneta & 2.316 & 4,53 \\
\hline Todos & Demais agentes causadores & 34.705 & 67,95 \\
\hline Agronegócio & Total & 51.071 & 100 \\
\hline
\end{tabular}

${ }^{1}$ Porcentagem calculada em relação ao total dos acidentes do agronegócio, com exceção do segmento de agrosserviços.

${ }^{2}$ Todos: segmentos de insumos, primário e agroindustrial.

Fonte: Autores com base em dados do OSST (2018).

A taxa incidência de acidentes de trabalho no agronegócio, para o período estudado, foi de 72,73 acidentes para cada 1000 trabalhadores. A Mesorregião Centro Oriental Paranaense teve a maior taxa, 97,31/1000, já a menor incidência ocorreu na Mesorregião Centro-Sul Paranaense com 47,5/1000 (Mapa 1). 
Mapa 1. Taxa de incidência de acidentes de trabalho nas mesorregiões do Paraná, 2012-2017.

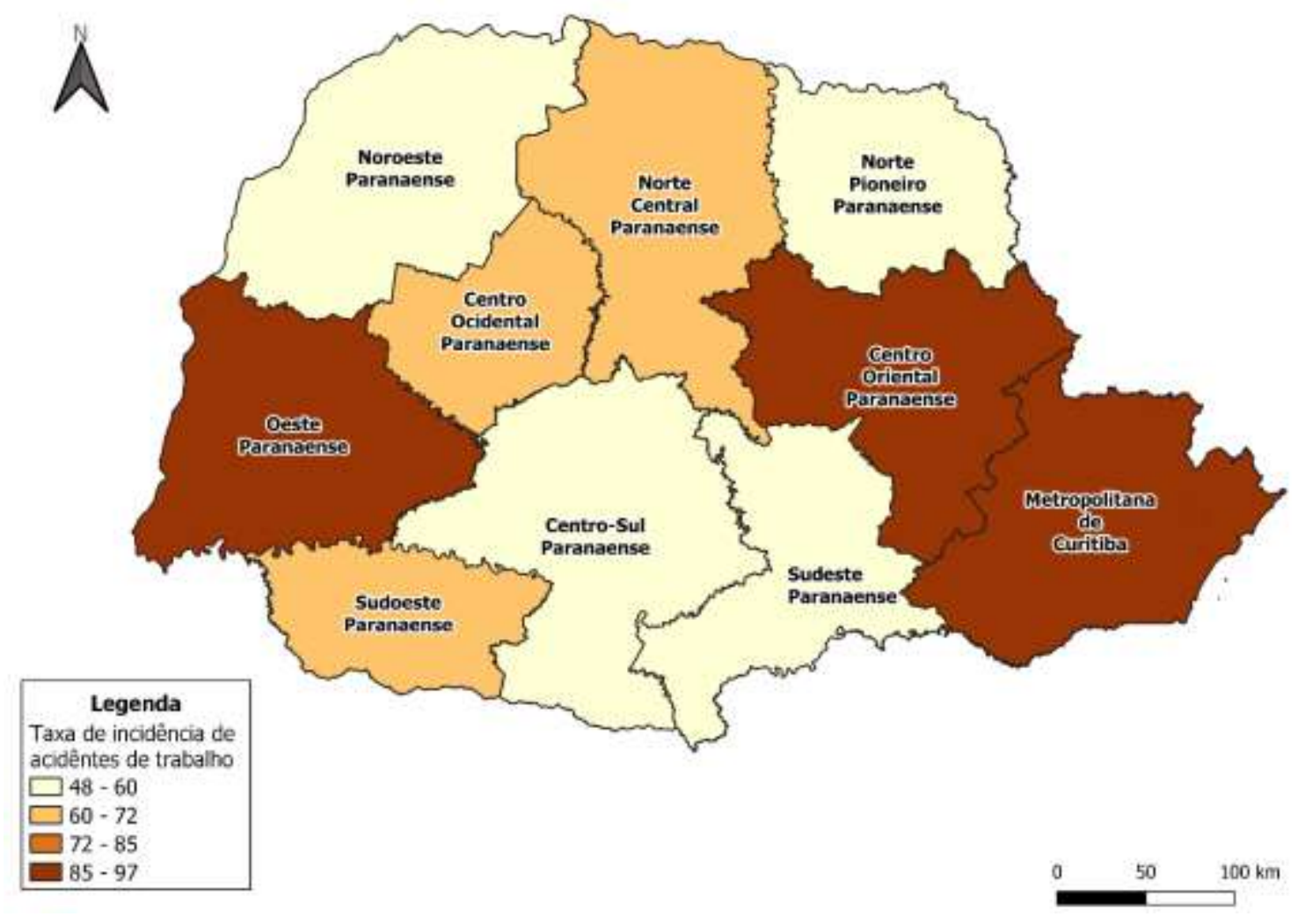

*Taxa de incidência calculada para 1000.

Fonte: Autores com base em dados do OSST 2018 e da RAIS 2020.

\section{Discussão}

A CAT é um importante instrumento que oferece informações previdenciárias, estatísticas e epidemiológicas de grande relevância e que pode proporcionar respaldo trabalhista aos empregados formais brasileiros (Ultramari et al., 2012). Assim, considera-se que o reconhecimento do ônus resultante dos 51.071 acidentes de trabalho ocorridos no processo produtivo dos segmentos de insumos, primário e agroindustrial, é um importante avanço para a busca da redução dos indicadores destes agravos e para identificar as vulnerabilidades ocupacionais do setor.

Porém, salienta-se que a CAT é de caráter obrigatório apenas para os trabalhadores formais, somado a isso há o fato de que muitas empresas registram apenas acidentes graves ou que geram afastamento superior a 15 dias. Existem ainda subnotificações, que causam problemas para identificação dos agravos relacionados ao trabalho (Ultramari et al., 2012). O OSST (2018), estima que em 2018, no Paraná, 21,8\% dos casos de acidentes de trabalho foram subnotificados.

Entre as CAT registradas, identificaram-se 254 óbitos. Houve prevalência de óbitos no sexo masculino 92,91\% e em trabalhadores com idade entre 26 e 35 anos, 28,34\%. A maioria destas mortes, 38, 33\%, decorreu de lesões múltiplas, e os principais agentes causadores foram os veículos 38\% e as motocicletas 7,08\%. Segundo Lee et al. (2012), o sexo e a idade associaram-se aos acidentes de trabalho fatais na agropecuária da Coréia do Sul. Sobre aos principais agentes causadores, estudos mostraram que o uso de veículos (Brière, Chevalier \& Imbernon, 2010; Mora et al., 2011), máquinas e equipamentos agrícolas (Lee et al., 2012), animais e queda de altura (Brière, Chevalier \& Imbernon, 2010; Mora et al., 2011) forma os mais comumente observados.

A maior taxa de mortalidade encontrada foi para o ano de 2012, em que ocorreram 7,29 óbitos a cada 100 mil ocupados. Este dado é semelhante aos encontrados na literatura. Ferreira-de-Sousa e Santana (2016) estimaram o coeficiente de mortalidade 
por acidentes de trabalho na agropecuária no Brasil, no ano de 2010 e, identificaram 7,3 mortes a cada 100 mil trabalhadores. Já na agropecuária da União Europeia, nesse mesmo ano, este coeficiente foi de 4,5/100.000 (European Commission, 2013).

Foi identificada redução do número de acidentes de trabalho em todos os segmentos analisados. Esta queda pode estar relacionada com a diminuição do número da população ocupada e com o novo ciclo de inovação tecnológica do setor. O total de trabalhadores paranaenses formalmente ocupados nos segmentos de insumos, primário e agroindustrial reduziu 12,59\% entre 2012 e 2018, passando de 739.970 ocupados para 556.774 (Ministério do Trabalho, 2020). O segmento da agroindústria contribuiu expressivamente com uma taxa de $77,07 \%$ para a redução de empregos formais durante o período, seguidos pelos segmentos primário e insumos, com taxa de participação da contribuição de 18,24\% e 4,70\%, respectivamente (Ministério do Trabalho, 2020). Já a redução brasileira foi de 19,60 milhões para 18,23 milhões de profissionais, representando uma redução de 7,02\% (Souza Jr. et al., 2020). Segundo Souza Jr et al. (2020), estas quedas evidenciaram-se nos anos 2000 e foram verificadas também no Rio Grande do Sul (Finamore \& Montoya, 2003), em Minas Gerais (Castro et al., 2017) e na região do Matopiba (Serigati et al., 2017).

De acordo com Manyika et al. (2017), é possível que até o ano de 2055 a produção de alimentos seja abalada pela a revolução digital e automação, o que poderá ocasionar impactos nas funções atualmente desempenhadas, na geração de empregos e nos acidentes de trabalho. Staduto et al. (2004) sustentam que a composição da mão de obra se modifica em função do novo ciclo de inovação tecnológica, poupador de mão de obra e minimizador de custos. Ainda Sakamoto (2014), infere que a diminuição da mão de obra no setor, relaciona-se também a inviabilidade de pequenos estabelecimentos rurais frente a este processo, somado a intensificação e concentração da produção e a existência de oportunidades em áreas urbanas em certas regiões.

Já a taxa de incidência de acidentes de trabalho no agronegócio paranaense, foi de 72,73 acidentes para cada 1000 trabalhadores, entre 2012 e 2018, maior que a taxa identificada em Ontário no Canadá, que registrou 58 acidentes para cada 1000 trabalhadores (Pickett et al., 1995), e a da Finlândia 62/1000 (Karttunen \& Rautiainen, 2013). Ao analisar mesorregiões específicas, a situação se torna mais preocupante, uma vez que o Centro Oriental apresentou taxa de 97,31/1000, o Oeste 89,8/1000 e a região Metropolitana de Curitiba 86,03/1000, indicando vulnerabilidade frente a temática analisada. Estes achados podem estar relacionados também com a composição da população ocupada nestes locais, tendo em vista que entre 2012 e 2017 as regiões Metropolitana de Curitiba e Oeste estiveram entre as que mais ocuparam mão de obra nos segmentos analisados (Ministério do Trabalho, 2020). Ademais, o segmento agroindustrial, no qual ocorreram 82,45\% dos acidentes, também concentrou a maioria dos trabalhadores destas mesorregiões, durante o período analisado (Ministério do Trabalho, 2020).

Quanto ao perfil dos acidentes, a evidência de maior proporção destes agravos envolvendo homens jovens corrobora com outros achados de estudos nacionais (Begnini \& Almeida, 2015; Mascarenhas et al., 2015; Moreira et al., 2015; Scussiato et al., 2013). Isso pode estar relacionado com o fato de que a população masculina, jovem e em idade produtiva, geralmente desempenha serviços mais perigosos e que demandam maior força física, o que demonstra a exigência de se promover ações de prevenção de acidentes com esta população (Mascarenhas et al., 2015; Scussiato et al., 2013).

A prevalência percentual de acidentes típicos, nos segmentos do agronegócio, em detrimento das doenças de trabalho também está alinhada com a literatura (Teixeira \& Freitas, 2005; Vasconcellos et al., 2009). Esse fato, pode ocorrer em função da visibilidade da lesão que os acidentes típicos causam, o qual possibilita o estabelecimento do nexo causal com o ambiente de trabalho, diferentemente das doenças do trabalho, em que há dificuldade de vincular o agravo a ocupação exercida (Teixeira \& Freitas, 2005).

No Paraná, o abate de suínos, aves e outros pequenos animais foi a atividade econômica com maior número de registro de CAT, com 12.923 casos de acidentes comunicados, 25,3\% do total. Esse dado se relaciona com um processo produtivo em que as ferramentas de trabalho podem acrescentar riscos e impactar no perfil de acidentes de trabalho. Corroborando, a ocupação 
mais vulnerável, foi a de alimentador de linha de produção, que registrou $10.230(20,4 \%)$ acidentes, enquanto as máquinas estiveram entre as ferramentas que mais causaram acidentes de trabalho, com 3.360 ocorrências, $6,58 \%$ do total.

Os frigoríficos são os locais de maior concentração desta atividade econômica. Vasconcellos et al. (2009) relatam que nestes ambientes o trabalho é especializado, as atividades se realizam ao longo de linhas de produção, em trilhos onde a matéria prima se desloca de modo que cada trabalhador realiza apenas uma função. Essas tarefas, como o corte, por exemplo, podem exigir entre 10 mil a 20 mil movimentos repetitivos diários (Vasconcellos et al., 2009).

Atividades econômicas relacionadas com a madeira também tiveram destaque neste trabalho. $\mathrm{O}$ desdobramento de madeira, a fabricação de móveis com predominância de madeira, a fabricação de madeira laminada e de chapas de madeira somam juntas 7.336 acidentes, o que representam 14,37\% das CAT dos segmentos do agronegócio. Ultramari et al. (2012) chamam a atenção para o fato de que pode haver locais onde a atividades de derrubada, desdobramento e até mesmo fabricação de móveis e carpintaria pode ser exercida de maneira rudimentar, inserida no meio rural e longe do acesso a serviços de saúde e informações, o que pode elevar a possibilidade de acidentes. No Paraná ocorreram 2.803 (5,49\%) acidentes com madeira como agente causador. A madeira, é considerada um produto pesado e que exige grande esforço do trabalhador durante o manuseio, podendo oferecer riscos ocupacionais (Lipscomb et al., 2009).

Outra atividade econômica importante neste trabalho foi a fabricação de açúcar em bruto, que registrou 2.626 CAT (5,14\%). Trabalhadores da cultura de cana-de-açúcar sofreram 3.099 (5,14\%) acidentes. Segundo Rumin e Schmidt (2008), as condições de trabalho na cultura e na indústria da cana-de-açúcar expõem os trabalhadores a exigências posturais, dispêndio de força muscular excessiva e riscos de acidentes relacionados a ferramentas do trabalho, como facões e equipamentos industriais.

Os achados revelam que houve um número grande de acidentes com facas e facão como agentes causadores, foram $4.782(9,36 \%)$ acidentes relatados, o que pode se relacionar com o fato que estes são instrumentos típicos de algumas atividades como a cultura de cana-de-açúcar e o abate de animais. Já as máquinas, veículos e motocicletas entraram neste cenário de forma expressiva, sendo responsáveis por 3.360 (6,58\%), $3.105(6,08 \%)$ e $2.316(4,53 \%)$ acidentes, respectivamente. Destaca-se que a OIT indica que entre as atividades agrícolas, as realizadas com máquinas, principalmente tratores e implementos, oferecem um elevado percentual de risco, sendo que um em cada três acidentes com esses equipamentos, culmina em incapacidade permanente para o trabalhador (Queiroz et al., 2008).

Os achados sobre as lesões mais frequentes, em que houve prevalência de cortes com 13.521 (26,47\%), fraturas 9.062 (17,74\%) e contusões 8.941 (17,51\%), corroboram com os resultados alcançados por Alves e Guimarães (2012) que, ao avaliar os acidentes e os adoecimentos em atividades rurais em Unaí, Minas Gerais, também verificaram que as lacerações, distensões, torções e fraturas nos membros superiores são os principais tipos de acidentes. Enquanto isso, a maioria das lesões, que deramse no âmbito do agronegócio paranaense, ocorreram nos dedos, 15.907 (31,15\%), nas mãos, 4.041 (7,91\%) e nos pés, 2.824 (5,53\%). Estudo realizado nas microrregiões de Chapecó, Concórdia e Xanxerê, no Oeste do Estado de Santa Catarina, identificou que as partes do corpo mais atingidas foram o punho e a mão, seguida por joelho e perna e tornozelo e pé (Begnini \& Almeida, 2015). Assim, ressalta-se a necessidade de ações de segurança do trabalhado, com ênfase no uso correto de Equipamentos de Proteção Individual (EPI), pensadas na proteção destas partes do corpo contra as principais lesões ocorridas.

A teoria do risco de acidente do trabalho indica que os principais agentes de risco ocupacional são os físicos, mecânicos, biológicos, ergonômicos e psicossociais (Teixeira \& Freitas, 2005). No agronegócio paranaense, são distintas as realidades vivenciadas pelos trabalhadores, existe convívio do uso de alta tecnologia agrária e industrial, em que o desenvolvimento tecnológico ampliou o uso de máquinas, veículos e outros equipamentos rurais e industriais, modificando os fatores de risco ocupacionais (Fernandes \& Silva, 2019), com condições simples de plantio e colheita, que possuem baixa capacitação tecnológica.

Ainda, reforçamos a importância de bando de dados como o do OSST para pesquisas relacionadas com o tema deste 
estudo. Fontes de dados secundárias permitem o acesso a amplas amostras, são de baixo custo e possuem boa flexibilidade metodológica.

\section{Considerações Finais}

Este estudo reforça a importância da análise dos acidentes de trabalho no agronegócio paranaense. A taxa de incidência foi de 72,73 acidentes para cada 1000 trabalhadores. As mesorregiões mais vulneráveis foram a Centro Oriental com 97,31/1000, a Oeste com 89,8/1000 e a Metropolitana de Curitiba 86,03/1000.

O estudo inova, pois, além de abranger todo o Estado do Paraná, também faz um detalhamento dos acidentes por segmentos do agronegócio, o que proporcionou a ampliação do conhecimento sobre os acidentes de trabalho no setor. Houve destaque para as CAT registadas no abate de suínos, aves e outros pequenos animais, com 12.923 (25,30\%) acidentes e para as atividades econômicas relacionadas ao processamento da madeira, em que ocorreram 7.336 (14,37\%) registros de CAT. A ocupação mais vulnerável foi o alimentador de linha de produção, com 10.230 (20,04\%) acidentados. As lesões mais prevalentes foram os cortes, lacerações, feridas contusas e puncturas com 13.521 (26,47\%) casos. A parte do corpo mais atingida foram os dedos, que registraram $15.907(31,15 \%)$ casos. As facas e fações representaram os principais agentes causadores, sendo responsáveis por $4.782(9,36 \%)$ acidentes.

Contudo, nossa seleção não assegura que os trabalhadores compartilhem do mesmo ambiente, o que pode ser considerada uma limitação do estudo. A qualidade e completude das informações das CAT, na qual se verificaram dados preenchidos de maneira incompleta e, o fato que as CAT são obrigatórias apenas para acidentes envolvendo a população que possui carteira assinada, também podem ser consideradas limitações.

Sugerem-se novos estudos, focados nas atividades econômicas e ocupações vulneráveis, a fim de estender o monitoramento dos fatores de risco das atividades ligadas ao agronegócio.

\section{Agradecimentos}

Agradecimentos pelo incentivo a pesquisa ao Programa de Pós Graduação em Desenvolvimento Regional e Agronegócio da Universidade Estadual do Oeste do Paraná.

\section{Referências}

Alves, R. A., \& Guimarães, M. C. (2012). De que sofrem os trabalhadores rurais? Análise dos principais motivos de acidentes e adoecimentos nas atividades rurais. Informe Gepec, 16(2), 39-56. http://e-revista.unioeste.br/index.php/gepec/article/download/5563/6988.

Begnini, S., \& Almeida, L. E. D. F. (2015). Acidentes de trabalho no meio rural: perfil do trabalhador acidentado em Santa Catarina, Brasil. Revista Eletrônica Gestão \& Saúde, 6(3), 2538-2552. https://periodicos.unb.br/index.php/rgs/article/view/3128/2814.

Brière, J., Chevalier, A., \& Imbernon, E. (2010). Surveillance of fatal occupational injuries in France: 2002-2004. American journal of industrial medicine, 53(11), 1109-1118. https://doi.org/10.1002/ajim.20874.

Castro, N. R., Barros, G. S. C., Almeida, A. N., Gilio, L., \& Morais, A. C. P. (2017). Mercado de trabalho e rendimentos no agronegócio de Minas Gerais. Revista de Economia e Agronegócio, 15(3), 386-405. https://doi.org/10.25070/rea.v15i3.490.

CEPEA - Centro De Estudos Avançados Em Economia Aplicada. (2017). Metodologia PIB do Agronegócio Brasileiro: Base e Evolução. https://www.cepea.esalq.usp.br/upload/kceditor/files/Metodologia\%20PIB_divulga\%C3\%A7\%C3\%A3o.pdf.

Chagas, A. M. R., Salim, C. A., \& Servo, L. M. S., (Orgs.). (2012). Saúde e segurança no trabalho no Brasil: aspectos institucionais, sistemas de informação e indicadores (2a ed.): Fundacentro. Recuperado de https://www.ipea.gov.br/portal/images/stories/PDFs/livros/livros/livro_saudenotrabalho.pdf.

European Commission. (2013). European social statistics: 2013 edition. Luxembourg: Publications Office of the European Union. https://ec.europa.eu/eurostat/documents/3930297/5968986/KS-FP-13-001-EN.PDF.pdf/6952d836-7125-4ff5-a153-6ab1778bd4da?t=1415007731000.

Felema, J., Raiher, A. P., \& Ferreira, C. R. (2013). Agropecuária brasileira: desempenho regional e determinantes de produtividade. Revista de Economia $e$ Sociologia Rural, 51 (3), 555-573. https://dx.doi.org/10.1590/S0103-20032013000300008. 
Fernandes, G. A., \& Silva, L. F. (2019). Factors associated with work accidents in a rural area in Minas Gerais, Brazil. Revista brasileira de medicina do trabalho, 17(3), 378-386. https://doi.org/10.5327/Z1679443520190318.

Ferreira-de-Sousa, F. N., \& Santana, V. S. (2016). Mortalidade por acidentes de trabalho entre trabalhadores da agropecuária no Brasil, 2000-2010. Cadernos de Saúde Pública, 32(4), e00071914. https://dx.doi.org/10.1590/0102-311X00071914.

Finamore, E. B., \& Montoya, M. A. (2003). PIB, tributos, emprego, salários e saldo comercial no agronegócio gaúcho. Ensaios FEE, 24(1), 93-126.

Governo Federal. (2017). Ministério da Agricultura, Pecuária e Abastecimento. Valor Bruto da Produção (VBP) 2017. Brasília. https://www.gov.br/agricultura/pt-br/assuntos/politica-agricola/valor-bruto-da-producao-agropecuaria-vbp.

Instituto Brasileiro de Geografia e Estatística. (2019). SIDRA. Censo Agropecuário 2017: Resultados Definitivos. Rio de Janeiro. https://sidra.ibge.gov.br/pesquisa/censo-agropecuario/censo-agropecuario-2017.

Instituto Brasileiro de Geografia e Estatística. (2020). SIDRA. Estimativas populacionais $2020 . \quad$ Rio de Janeiro. https://sidra.ibge.gov.br/pesquisa/estimapop/tabelas.

Karttunen, J. P., \& Rautiainen, R. H. (2013). Distribution and characteristics of occupational injuries and diseases among farmers: a retrospective analysis of workers' compensation claims. American journal of industrial medicine, 56(8), 856-869. https://doi.org/10.1002/ajim.22194.

Lee, S. J., Kim, I., Ryou, H., Lee, K. S., \& Kwon, Y. J. (2012). Work-related injuries and fatalities among farmers in South Korea. American journal of industrial medicine, 55(1), 76-83. https://doi.org/10.1002/ajim.21016.

Lipscomb, H. J., Dement, J. M., Silverstein, B., Cameron, W., \& Glazner, J. E. (2009). Compensation costs of work-related back disorders among union carpenters, Washington State 1989-2003. American journal of industrial medicine, 52(8), 587-595. https://doi.org/10.1002/ajim.20715.

Manyika, J., Chui, M., Miremadi, M., Bughin, J., George, K., Willmott, P., \& Dewhurst, M. (2017). A future that works: Automation, employment, and productivity. New York: McKinsey Global Institute. https://www.mckinsey.com/ /media/mckinsey/featured\%20insights/Digital\%20Disruption/Harne ssing\% 20a utomation $\% 20$ for $\% 20 \mathrm{a} \% 20$ future $\% 20$ that $\% 20$ works/MGI-A-future-that-works-Executive-summary.ashx.

Mascarenhas, M. D. M., Freitas, M. G., Monteiro, R. A., Silva, M. M. A., Malta, D. C., \& Gómez, C. M. (2015). Emergency room visits for work-related injuries: characteristics and associated factors - Capitals and the Federal District, Brazil, 2011. Ciência \& Saúde Coletiva, 20(3), 667-678. https://doi.org/10.1590/141381232015203.16842014 .

Ministério da Economia. (2020). Secretaria Especial de Previdência e Trabalho. Anuário Estatístico da Previdência Social 2013: Subseção A - Acidentes do Trabalho. Brasília. https://www.gov.br/previdencia/pt-br/assuntos/saude-e-seguranca-do-trabalhador/dados-de-acidentes-do-trabalho/aeat-2013/subsecao-a.

Ministério do Trabalho. (2020). PDET - Programa de Disseminação das Estatísticas do Trabalho. Microdados RAIS e CAGED. Brasília. http://pdet.mte.gov.br/microdados-rais-e-caged?_ga=2.76313365.1278749075.1612490536-1284000383.1612490536.

Mora, A. M., Mora-Mora, M. G., Partanen, T., \& Wesseling, C. (2011). Registration of fatal occupational injuries in Costa Rica, 2005-2006. International journal of occupational and environmental health, 17(3), 243-250. https://www.tandfonline.com/doi/abs/10.1179/107735211799041913

Moreira, J. P. L., Oliveira, B. L. C. A., Muzi, C. D., Cunha, C. L. F., Brito, A. S., \& Luiz, R. R. (2015). A saúde dos trabalhadores da atividade rural no Brasil. Cadernos de Saúde Pública, 31(8), 1698-1708. https://doi.org/10.1590/0102-311X00105114.

OSST - Observatório Digital de Saúde e Segurança no Trabalho. (2018). Segurança e Saúde no Trabalho. Recuperado de https://smartlabbr.org/sst.

Pickett, W., Brison, R. J., Niezgoda, H., \& Chipman, M. L. (1995). Nonfatal farm injuries in Ontario: a population-based survey. Accident Analysis and Prevention, 27(4), 425-433. https://doi.org/10.1016/0001-4575(94)00080-6.

Prestes, A. F., Corte, G. M. D., Cattelan, R., \& Moraes, M. L. (2018). Impacto do agronegócio no desenvolvimento sustentável paranaense. Revista de política agrícola, 27(3), 114-130. https://seer.sede.embrapa.br/index.php/RPA/article/view/1448/pdf.

Queiroz, M. T. A, Cotta, S. C., Saliba, G. A., Furtado, B. M. B., \& Costa, K. A. (2008). Análise dos acidentes de trabalho relativos às atividades agropecuárias no colar metropolitano da região do Vale do Aço no Período de 2002 a 2007. Anais do V Simpósio de Excelência em Gestão e Tecnologia - SEGeT. Resende, RJ. https://www.aedb.br/seget/arquivos/artigos08/3_Acidentes\%20na\%20area\%20rura1\%20-\%20SEGET\%20F.pdf.

Rumin, C. R., \& Schmidt, M. L. G. (2008). Influências das condições e organização do trabalho de uma indústria de transformação de cana-de-açúcar na ocorrência de acidentes de trabalho. Saúde e Sociedade, 17(4), 56-67. https://doi.org/10.1590/S0104-12902008000400007.

Sakamoto, C. S. (2014). Mudanças na composição das famílias e impactos na distribuição de rendimentos: um comparativo entre áreas rurais e urbanas no Brasil. Dissertação de Mestrado, Universidade Estadual de Campinas, Instituto de Economia, Campinas. http://repositorio.unicamp.br/jspui/bitstream/REPOSIP/286500/1/Sakamoto_CamilaStrobl_M.pdf.

Serigati, F., Rodrigues, R. M., Possamai, R., \& Vieira Filho, J. E. R. (2017). Texto para Discussão (TD) 2277: O mercado de trabalho na fronteira do agronegócio: quanto a dinâmica no Matopiba difere das regiões mais tradicionais? Instituto de Pesquisa Econômica Aplicada - Brasília: Ipea. https://www.ipea.gov.br/portal/images/stories/PDFs/TDs/td_2277.pdf.

Scussiato, L. A., Sarquis, L. M. M., Kirchhof, A. L. C., \& Kalinke, L. P. (2013). Epidemiological profile of serious accidents at work in the State of Paraná, Brazil, 2007-2010. Epidemiologia e Serviços de Saúde, 22(4), 621-630. https://dx.doi.org/10.5123/S1679-49742013000400008.

Souza Jr., M. L., Castro, N. R., Gilio, L., Morais, A. C. P., \& Barros, G. S. C. (2020). Mercado de trabalho do agronegócio no centro-oeste: a importância do setor para o dinamismo regional. Revista de Economia e Agronegócio, 18(1). https://doi.org/10.25070/rea.v18i1.8426.

Staduto, J. A. R., Shikida, P. F. A., \& Bacha, C. J. C. (2004). Alteração na composição da mão de obra assalariada na agropecuária brasileira. Revista Agricultura em São Paulo, 51(2), 57-70. http://www.iea.sp.gov.br/out/publicacoes/pdf/asp-2-04-5.pdf. 
Research, Society and Development, v. 10, n. 2, e35110212710, 2021

(CC BY 4.0) | ISSN 2525-3409 | DOI: http://dx.doi.org/10.33448/rsd-v10i2.12710

Teixeira, M. L. P, \& Freitas, R. M. V. (2005). Acidentes no campo: perfil problemático. Revista Proteção, 160, 66-75.

Ultramari, A. V., Silva, A. M. C., \& Pignati, W. A. (2012). Ambiente de trabalho: influência da produção florestal sobre os acidentes do trabalho no Estado de Mato Grosso. Caderno de Saúde Coletiva, 20(1), 25-31. http://www.cadernos.iesc.ufrj.br/cadernos/images/csc/2012_1/artigos/CSC_v20n1_25-31.pdf.

Vasconcellos, M. C., Pignatti, M. G., \& Pignati, W. A. (2009). Emprego e acidentes de trabalho na indústria frigorífica em áreas de expansão do agronegócio, Mato Grosso, Brasil. Saúde e Sociedade, 18(4), 662-672. https://dx.doi.org/10.1590/S0104-12902009000400010. 